\title{
EHMTI-0090. Botox in the prevention of chronic migraine; comparing NICE criteria versus hull criteria for evaluating responder rate
}

\author{
H Zafar ${ }^{*}$, M Khalil, F Ahmed \\ From 4th European Headache and Migraine Trust International Congress: EHMTIC 2014 \\ Copenhagen, Denmark. 18-21 September 2014
}

\section{Background}

Chronic migraine (CM) affects $2 \%$ of the population and Botox is the only licensed treatment for prevention of adult patients with CM.

In the UK, National Institute for Clinical Excellence (NICE) approved its use on the National Health Service (NHS) provided patients had failed three preventive medications and appropriately addressed for medication overuse.

NICE defines responder with $30 \%$ reduction in headache days without emphasis on severity of headache or number of migraine days.

We developed Hull Criteria that defines responder as one with either:

$50 \%$ reduction in either

Headache days

Or migraine days

An increment in crystal clear days twice that of baseline

\section{Objectives}

To compare the outcomes of patients receiving Botox treatment in CM according to NICE and Hull Criteria.

\section{Results}

Out of a cohort of 357 patients having received a total of 858 cycles, we analysed 151 patients who had received two treatment cycles as recommended by NICE.

A Significant number of patients who responded with Hull Criteria did not satisfy NICE criteria and were denied treatment.

\section{Discussion}

We recommend that severity of headache and number of migraine days must be taken in to account in evaluating response rate to Botox.

NICE criteria should include reduction in migraine days in addition to headache days.

Published: 18 September 2014

doi:10.1186/1129-2377-15-S1-G2

Cite this article as: Zafar et al:: EHMTI-0090. Botox in the prevention of chronic migraine; comparing NICE criteria versus hull criteria for evaluating responder rate. The Journal of Headache and Pain 201415 (Suppl 1):G2.

\section{Method}

Adult patients with $\mathrm{CM}$ attending the Hull migraine clinic were offered Botox based on clinical needs and maintained a headache diary.

Data were extracted for headache, migraine, and headache-free days

Responder rate was assessed applying Hull and NICE criteria. 